\title{
Physics Prospects with GlueX
}

\author{
A. Somov \\ ( on behalf of the GlueX Collaboration)
}

Thomas Jefferson National Accelerator Facility, Newport News, Virginia 23606, USA

\begin{abstract}
The new experiment GlueX is currently being constructed at Jefferson Lab. The experiment was designed to search for hybrid mesons with exotic-quantum-numbers using a beam of linearly polarized photons incident on a liquid hydrogen target. We will discuss the discovery potential of the GlueX experiment and briefly overview its physics program.

Keywords: GlueX experiment, hybrid mesons

PACS: <12.38.Qk, 25.20.Lj, 29.27.Hj, 13.60.-r
\end{abstract}

\section{INTRODUCTION}

Understanding the role of gluons in the confinement of quarks in quantum chromodynamics (QCD) is one of the most tantalizing topics in modern particle physics to be explored. Perhaps the clearest way would be the experimental observations of the quark/gluon states whose quantum numbers $\left(J^{P C}\right)$ are forbidden by Quark Model and comparison their properties with detailed QCD calculations. Such states could be glueballs, multi-quark molecules $(q \bar{q} q \bar{q})$, or socalled hybrid mesons $(q \bar{q} g)$, where $g$ represents an excitation of the gluonic field binding the $q \bar{q}$ system. Experimental observation of several hybrid meson candidates with exotic quantum numbers brought them into research focus of many theoreticians and stimulated further experimental search. The origin of exotic quantum numbers can be explained based on the so-called flux tube model [1], the model which has so far been successfully verified for heavy quarks using lattice QCD calculations [2]. In the flux tube model, the gluonic field, or the "flux tube" can have an angular momentum, $m$, which can be combined with the quantum numbers of a $q \bar{q}$ system. When the flux tube is in the ground state, $\mathrm{m}=0$, the fermion-antifermion system $(q \bar{q})$ can be characterized by specific quantum numbers, $J^{P C}$, corresponding to the conventional mesons, where $J$ is the total angular momentum, $\mathrm{P}$ is parity and $\mathrm{C}$ is $\mathrm{C}$-parity. If the flux tube is in the first excited state, $\mathrm{m}=1$, in addition to the quantum numbers of the normal mesons, the $q \bar{q} g$ system can have exotic quantum numbers, such as $J^{P C}=0^{+-}, 1^{-+}, 2^{+-}$. Each of these $J^{P C}$ states corresponds to a nonet of mesons. Recent lattice calculations predict masses of hybrid mesons with $J^{P C}=0^{+-}, 1^{-+}, 2^{+-}$to be between 1.8 $\mathrm{GeV}$ and $2.8 \mathrm{GeV}$ and $J^{P C}=1^{-+}$to be the lightest state. This predictions define the energy region for the experimental search of light hybrid mesons. Experimental observation of the nonets of the exotic-quantum-number mesons across several final states would certainly help in interpretation of the observed states and provide important consistency checks of theoretical models.

Exotic mesons have been extensively searched during the past few decades by several experiments using different beam particles and energies. Several experiments have reported on observing three exotic states $\pi_{1}(1400), \pi_{1}(1600)$, and $\pi_{1}(2015)$ with the quantum number $J^{P C}=1^{-+}$. The $\pi_{1}(1400)$ state has been seen by most experiments in $\eta \pi$ decay channel only. This does not agree with the expected decay modes of hybrid mesons. Furthermore, the $\pi_{1}(1400)$ mass is also smaller than the model predictions. The $\pi_{1}(1600)$ state is more consistent with the hybrid meson. It has been seen in $b_{1} \pi, f_{1} \pi$, and $\eta^{\prime} \pi$ final states by VES and E852 collaboration. However, the $\rho \pi$ decay channel is controversial. This decay mode had originally been seen by the E852 experiment but was ruled out by the later analysis. Meantime, the COMPASS collaboration confirmed the $\rho \pi$ decay. No $\pi_{1}(1600) \rightarrow \pi^{+} \pi^{-} \pi^{+}$signal has been observed by CLAS collaboration in photoproduction at about $6 \mathrm{GeV}$. The third hybrid meson candidate is $\pi_{1}(2015)$, whose observation was reported by the E852 experiment in $f_{1} \pi$ and $b_{1} \pi$ final states with limited statistics. Obviously, more data are needed to understand the nature of the discovered exotic states. More information on the current status of the meson spectroscopy can be found in [3]. Two new experiments dedicated to search for states with exotic quantum numbers are currently under construction: PANDA at GSI and GlueX at Jefferson Lab. Panda will perform a search for exotic states in $p \bar{p}$ annihilation while GlueX will use a beam of linearly polarized photons.

The production of exotic mesons is expected to be enhanced in $\gamma p$ interactions, where the experimental data is very limited. Naively, this can be explained by combining the quantum number of a beam photon $J^{P C}=1^{--}$, which, 


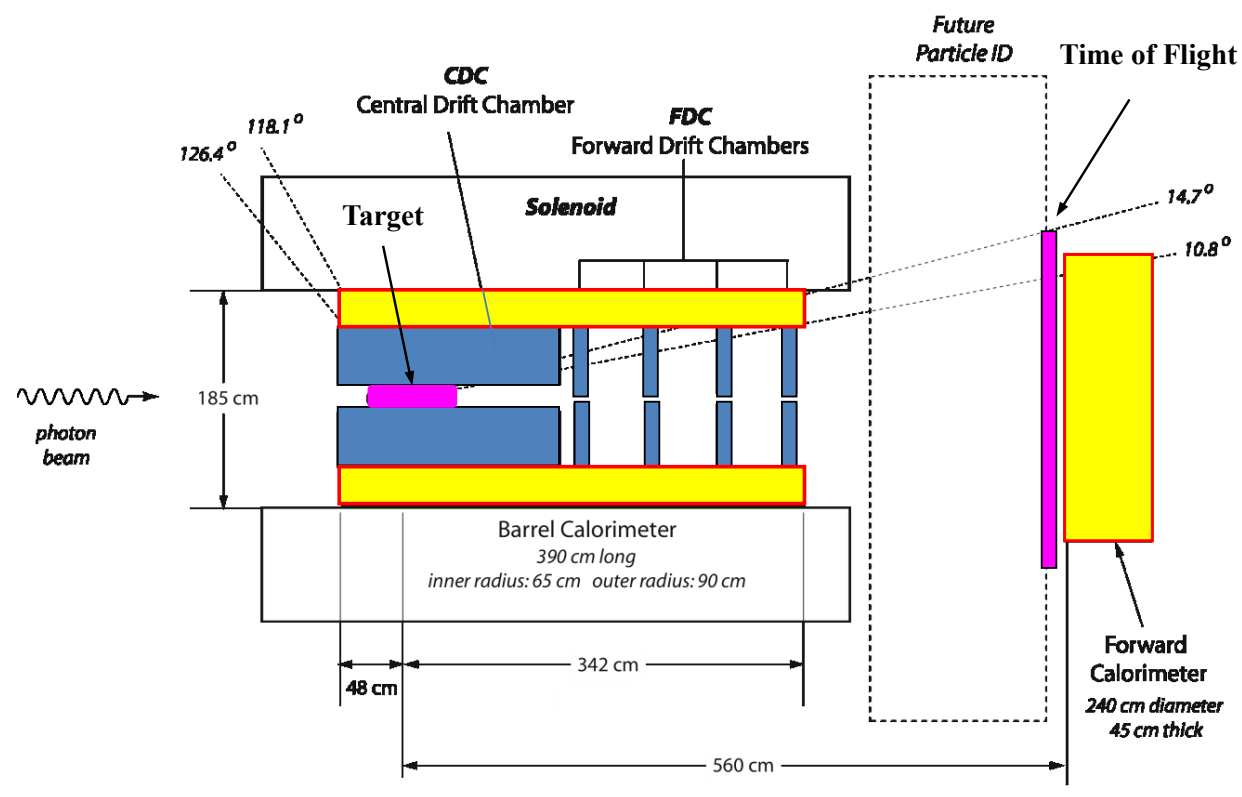

FIGURE 1. Layout of the GlueX detector.

according to the vector dominance model can be presented as a virtual $q \bar{q}$ pair with $L=0$ and $S=1$, with the quantum numbers of the excited flux tube, $1^{+-}$or $1^{-+}$. As a results, the states with exotic $J^{P C}$ can be directly formed. However, in the case of the $\pi$ beam, production of exotic mesons is expected to be suppressed because a spin flip of the quark is required. Large photoproduction cross-sections for exotic mesons are also predicted by recent lattice calculations of the radiative decay of charmonium states into hybrid mesons with exotic quantum numbers [4].

The upgrade of the CEBAF electron beam energy from $6 \mathrm{GeV}$ to $12 \mathrm{GeV}$ will provide a unique high-intensity beam of linearly polarized photons produced using a Bremsstrahlung technique. The GlueX experiment at Jefferson Lab has been designed to search for mesons with exotic-quantum-numbers using this beam. The GlueX experiment will collect a data sample a few order of magnitudes larger than all existing photoproduction data.

\section{GLUEX EXPERIMENT}

The photon beam used by the GlueX experiment will be produced via coherent Bremsstrahlung process by $12 \mathrm{GeV}$ electrons incident on a $20 \mu \mathrm{m}$ thick diamond radiator. In order to increase the fraction of linearly polarized photons in the energy region of interest between $8.4 \mathrm{GeV}$ and $9.1 \mathrm{GeV}$ (the coherent peak region), the photon beam will be passed through a collimator situated about $75 \mathrm{~m}$ downstream from the radiator. The collimated photons will subsequently be sent to the GlueX liquid hydrogen target. The photon beam energy of $\sim 9 \mathrm{GeV}$ will provide enough statistics to study the spectrum of exotic meson candidates up to about $2.8 \mathrm{GeV} / \mathrm{c}^{2}$. The photon energy will be determined with an accuracy of about $0.1 \%$ by measuring the energy of the electron after radiation. The GlueX detector will start operating using the flux of tagged photon of $10^{7} \mathrm{\gamma} / \mathrm{s}(8.4-9 \mathrm{GeV})$ on the target. Later, after installing a computer farm that will be used in the Level-3 trigger system, the beam intensity will be increased to $10^{8} \mathrm{\gamma} / \mathrm{s}$.

GlueX is a magnetic spectrometer, which will be located in the newly constructed experimental Hall-D at Jefferson Lab. The detector design has been optimized to provide almost a $4 \pi$ coverage for charged tracks and photons originating from the exotic meson candidates. The spectrometer layout is presented in Fig. 1. The central part of the detector will be situated inside the solenoid magnet operated at $2.2 \mathrm{~T}$ field. Charged tracks are reconstructed with the Central and Forward drift chambers, which cover the polar angle of the reconstructed tracks between $1^{\circ} \leq \theta \leq 155^{\circ}$ in the lab frame. Reconstruction of photons is performed using lead-scintillator (barrel) and lead glass (forward) calorimeters. The spectrometer will provide high detection efficiency for both charged tracks and photons and, therefore, will allow us to reconstruct exotic meson candidates in several final states. The angular acceptance of 
the detector is relatively large and uniform for most mesons of interest, which is particular important for the angular analysis of the observed mesons.

The quantum numbers $J^{P C}$ of the observed mesons will be determined using a partial wave analysis (PWA). The GlueX PWA software is being intensively tested using Monte Carlo simulations. An example of the detector capability to reconstruct an exotic $J^{P C}=1^{-+}$wave mixed with six non-exotic waves corresponding to $\gamma p \rightarrow \pi^{+} \pi^{-} \pi^{+} n$ events is presented in Fig.3. The exotic wave corresponds to $\pi_{1}(1600)$ meson, while other amplitudes were taken from the states such as $a_{1}, a_{2}$, and $\pi_{2}$. The fraction of the signal $\pi_{1}$ events constituted to about $2.5 \%$ of the total number of simulated events. Linear polarization of the beam photons provides important information for the PWA. In particular, the polarization introduces azimuthal dependence between the polarization vector and the production plane that depends on the parity of the exchanged particle in the photoproduction process and, therefore, allows one to constrain the final state quantum numbers. The polarization also leads to the azimuthal dependencies in the decay angle distributions.

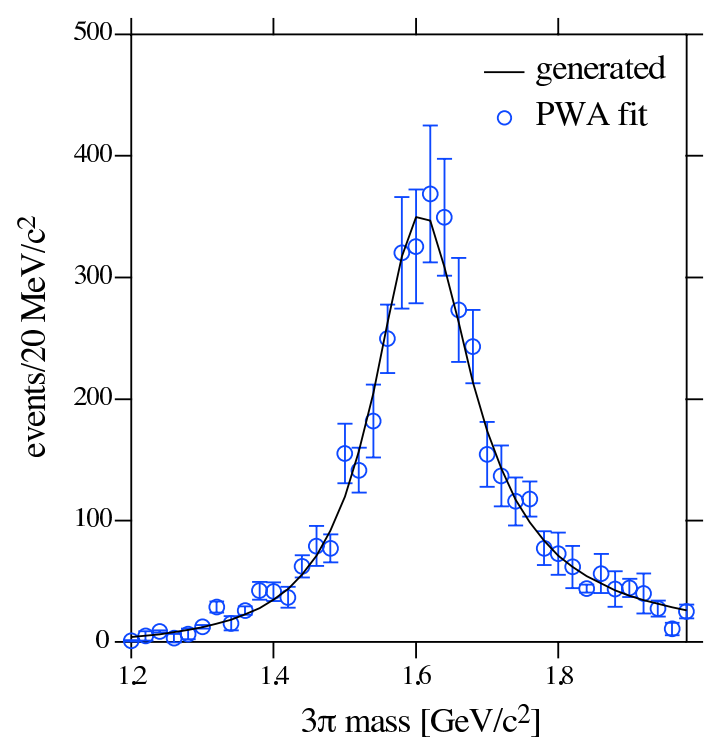

FIGURE 2. Results of the reconstruction of the $J^{P C}=1^{-+}\left(\pi_{1}(1600)\right)$ exotic wave mixed with six non-exotic waves corresponding to the $\gamma p \rightarrow \pi^{+} \pi^{-} \pi^{+} n$ events. The statistics correspond to a few days of running.

\section{OTHER PHYSICS TOPICS WITH GLUEX}

Being optimized for the meson spectroscopy, the GlueX detector is well-suited for studies of other physics topics such as photoproduction with nuclear targets and exclusive reactions at high-momentum transfer. The physics program of the GlueX is being continuously developing and is widely discussed [6]. Recently, a new experiment with the GlueX detector, PrimeX [7], has been approved by Jefferson Lab Program Advisory Committee. The experiment is aimed to measure the $\eta \rightarrow \gamma \gamma$ decay width via the Primakoff effect. The Primakoff effect represents production of mesons in interactions of the real beam photon with the virtual photon from the Coulomb field of the nucleus. The decay width can be extracted from the eta production angular distribution, which is presented in Fig.3. The measurements will allow to significantly improve the precision of the ratios of the light quark masses and the value of the $\eta-\eta^{\prime}$ mixing angle.

\section{OUTLOOK}

GlueX is a new experiment at Jefferson Lab. whose physics program is intended to improve our knowledge of strong interactions. The main goal of the experiment is to search for gluonic excitations in photoproduction. The experiment is expected to collect a data sample a few order of magnitudes larger than all existing photoproduction data. The physics 
topics of the experiment spans from light meson spectroscopy to Primakoff production of pseudoscalar mesons. The construction of the experiment has started in 2009 and the commissioning stage is expected to be finished in 2015 .

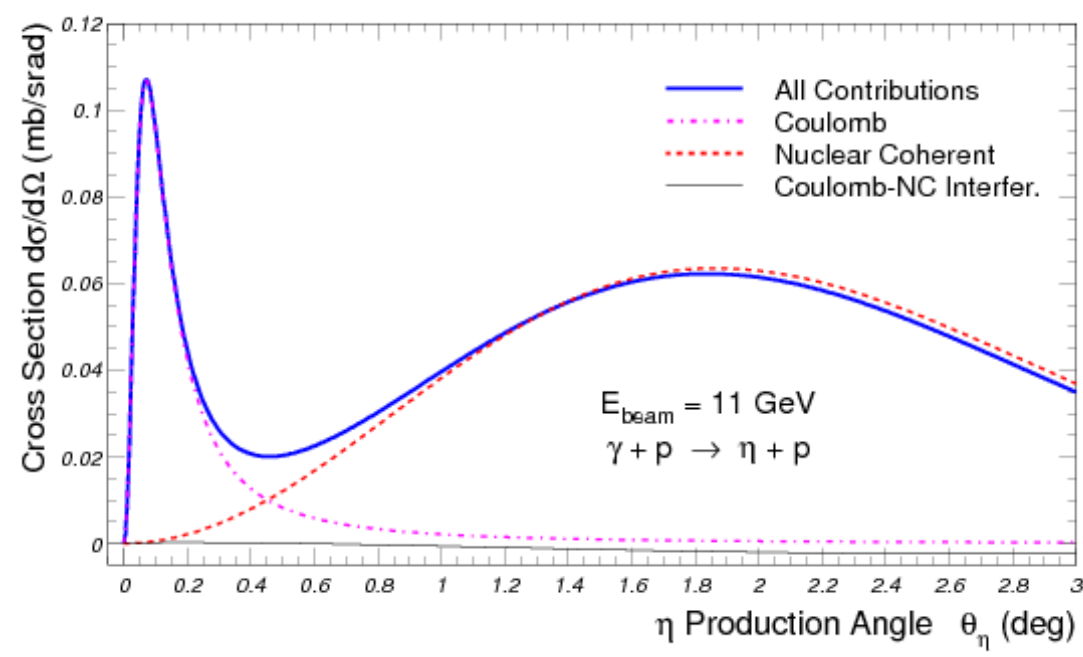

FIGURE 3. Differential cross sections for $\gamma p \rightarrow \eta p$ reaction. The curves show contribution from different processes: the dotdashed curve corresponds to the Primakoff production, the dashed curve curve corresponds to the nuclear coherent process, the small solid curve is the interference between the Primakoff and nuclear coherent, and the solid curve is the total.

\section{ACKNOWLEDGMENTS}

This work was supported by the US Department of Energy contract DE-AC05-06OR23177, under which Jefferson Science Associates, LLC operates Thomas Jefferson National Accelerator Facility. I would like to thank my colleagues from the GlueX collaboration for their help preparing this proceedings.

\section{REFERENCES}

1. N. Isgur and J. Paton, Phys.Rev.D 31, 2910, (1985),

N. Isgur, R. Kokoski and J.Paton, Phys.Rev.Lett. 54, 869, (1985).

2. G. Bali et al. [SESAM Collaboration], Nucl.Phys.Proc.Suppl. 63, 209, (1998).

3. C. A. Meyer, and Y. Van Haarlem, The Status of Exotic-quantum-number Mesons, arXiv:1004.5516, (2010),

E. Klempt and A. Zaitsev, Phys.Rep.454, 1, (2007).

4. J. J. Dudek, R. Edwards and C. E. Thomas, Phys.Rev.D 79, 094504 (2009).

5. J. J. Dudek, R. Edwards, and C. E. Thomas, Phys.Rev.D bf 79,094504 (2009).

6. Workshop on Photon-hadron physics with the GlueX detector, March 8, (2008), http://conferences.jlab.org/php2008/index.html.

7. A precision measurement of the $\eta$ radiative decay width via Primakoff effect, Proposal, Dec 2009. 